\title{
Phase I Study of Bortezomib Combined With Chemotherapy in Children With Relapsed Childhood Acute Lymphoblastic Leukemia (ALL): A Report From the Therapeutic Advances in Childhood Leukemia (TACL) Consortium
}

\author{
Yoav Messinger, $\mathrm{MD}^{1}{ }^{1 *}$ Paul Gaynon, $\mathrm{MD}^{2}{ }^{2}$ Elizabeth Raetz, $\mathrm{MD}^{3}$ Raymond Hutchinson, $\mathrm{MS}, \mathrm{MD}^{4}$ \\ Steven DuBois, $\mathrm{MD}^{5}$ Julia Glade-Bender, $\mathrm{MD}^{6}{ }^{6}$ Richard Sposto, $\mathrm{PhD}^{7}{ }^{7}$ Jeannette van der Giessen, $\mathrm{BA}^{7}$ \\ Elena Eckroth, $\mathrm{CCRC}^{7}$ and Bruce C. Bostrom, $\mathrm{MD}^{8}$
}

\begin{abstract}
Background. Outcomes remain poor for children after relapse of acute lymphoblastic leukemia (ALL), especially after early marrow relapse. Bortezomib is a proteasome inhibitor with in vitro synergy with corticosteroids and clinical activity in human lymphoid malignancies. Procedure. This is a Phase I study of escalating doses bortezomib administered days 1, 4, 8, and 11, added to 4-drug induction chemotherapy with vincristine, dexamethasone, pegylated L-asparaginase, and doxorubicin (VXLD) in children with relapsed ALL. Results. Ten patients were enrolled, five in first marrow relapse, and five in second relapse. Four patients were enrolled at dose level 1 (bortezomib $\left.1 \mathrm{mg} / \mathrm{m}^{2}\right)$. One patient was not evaluable for toxicity because of omitted dexamethasone doses. No dose-limiting toxicity (DLT) was observed. Six patients were enrolled at dose level 2 (bortezomib $1.3 \mathrm{mg} / \mathrm{m}^{2}$ ). One patient had dose-limiting hypophosphatemia and rhabdomyolysis after 1 dose of bortezomib, and died
\end{abstract}

from a diffuse zygomyces infection on day 17 . Five additional patients were enrolled with no subsequent DLTs. As planned, no further dose escalation was pursued. The regimen had predictable toxicity related to the chemotherapy drugs. Two patients had mild peripheral neuropathy (grades 1 and 2). Six of nine evaluable patients $(67 \%)$ achieved a complete response (CR), and one had a bone marrow CR with persistent central nervous system leukemia. Conclusions. The combination of bortezomib $\left(1.3 \mathrm{mg} / \mathrm{m}^{2}\right)$ with VXLD is active with acceptable toxicity in pretreated pediatric patients with relapsed ALL. We are expanding the $1.3 \mathrm{mg} / \mathrm{m}^{2}$ cohort for a phase II estimate of response. Study registered at ClinicalTrials.gov (http://clinicaltrials.gov/ct2/show/NCT00440726). Pediatr Blood Cancer 2010;55:254-259.

(c) 2010 Wiley-Liss, Inc.

Key words: ALL relapse; bortezomib; chemotherapy; phase I study

\section{INTRODUCTION}

Despite progress in childhood ALL, outcomes remain poor after relapse. Patients with very early marrow relapse (less than 18 months from diagnosis) have fared worse with a 5-year survival of about $15 \%$ [1]. In studies of patients with early marrow relapse, only those achieving complete remission (CR) after a single treatment block survived [2,3]. Children's Oncology Group (COG) studies showed a remission reinduction rate of about $70 \%$ after one treatment block for very early and early first marrow relapse [2,3]. Patients with very early relapsed ALL (less than 18 months from diagnosis) had low reinduction rate of $45 \%$ [3]. Review of the experience at Therapeutic Advances in Childhood Leukemia (TACL) institutions found a remission reinduction rate of $78 \%$ for very early and $86 \% \mathrm{CR}$ for early first marrow relapse [4]. Remissions were more difficult to achieve in children with multiple recurrences. The TACL group observed low remission rates of $44 \%, 27 \%$, and $12 \%$ for third, fourth, and further therapeutic attempts, respectively [4]. In that study disease-free survival (DFS) of patients in CR3 or above was only $15 \%$ at 5 years, indicating the poor outcome of multiple relapsed patients [4]. Since limited progress in the treatment of bone marrow relapsed ALL has been documented over the past 20 years [1] and outcomes remain poor, new therapeutic strategies for relapsed childhood leukemia are urgently needed.

The TACL consortium chose to study bortezomib as a new agent in this population based on promising preclinical activity and success in other hematopoietic malignancies. Bortezomib is a selective inhibitor of the ubiquitin proteasome pathway (UPP), which is an essential pathway for the degradation of most proteins in eukaryotic cells [5]. Important regulatory proteins affected by inhibition of the UPP system include NF- $\kappa$ B, p53, Bax, p27, and p21 [6]. Proteasome inhibition may sensitize malignant hematologic cells to apoptosis induced by both radiation and chemotherapy [7-9]. Bortezomib has received FDA approval for use in adults with multiple myeloma and relapsed NHL [10]. Preclinical studies did show some activity of single agent bortezomib in ALL [11]. Unfortunately, clinical studies showed very little activity of the single agent bortezomib in either adults [12] or children [13] with acute leukemia.

Bortezomib may have enhanced anti-leukemic activity when used in combination with chemotherapy. Horton et al. [14] have shown that bortezomib and dexamethasone have synergistic anti-leukemia interactions in vitro, and bortezomib had additive anti-leukemia effects when combined with asparaginase, vincristine, doxorubicin, or cytosine arabinoside. A multiply relapsed child with ALL had transient clinical response to bortezomib and

${ }^{1}$ Pediatric Hematology and Oncology, Children's Hospitals and Clinics of Minnesota, St. Paul, Minnesota; ${ }^{2}$ Division of Hematology/ Oncology, University of Southern California, Los Angeles, California; ${ }^{3}$ Department of Pediatrics, New York University School of Medicine, New York, New York; ${ }^{4}$ University of Michigan Medical School, Ann Arbor, Michigan; ${ }^{5}$ Department of Pediatrics, UCSF School of Medicine, San Francisco, California; ${ }^{6}$ Pediatric Oncology, Columbia University, New York, New York; ${ }^{7}$ Children's Center for Cancer and Blood Diseases, Children's Hospital Los Angeles, Los Angeles, California; ${ }^{8}$ Pediatric Hematology and Oncology, Children's Hospitals and Clinics of Minnesota, Minneapolis, Minnesota

Conflict of interest: Paul Gaynon, MD: honoraria from Enzon, Allos, and Sanofi Aventis, Chair of data monitoring committee (dasatinib) for Bristol Meyers. No one has any conflict of interest with Millennium Pharmaceuticals, or reports any other conflict of interest. Millennium Pharmaceuticals did not sponsor this study, or provide funds or drug for it.

*Correspondence to: Yoav Messinger, Pediatric Hematology and Oncology, Children's Hospitals and Clinics of Minnesota, 345 N. Smith Avenue, St. Paul, MN 55102. E-mail: yoav.messinger@childrensmn.org

Received 6 November 2009; Accepted 6 January 2010 
dexamethasone [15]. In a phase I adult acute myeloid leukemia (AML), Attar et al. [16] have shown that the combination of bortezomib with idarubicin and cytosine arabinoside resulted in $61 \%$ CR's and an additional $10 \%$ with CR's but incomplete platelet recovery (CRp).

Based on these studies, we added bortezomib to a standard chemotherapy platform of vincristine, dexamethasone, pegylated L-asparaginase (PEG-asparaginase), and doxorubicin (VXLD) platform. In a phase I/II study, we sought to determine whether the previously defined single agent dose of bortezomib is tolerable in this context and to estimate a phase II response rate in second relapse patients. The phase I part of this study is reported here.

\section{PATIENTS AND METHODS}

This study was approved by the Institutional Review Boards (IRBs) of all participating TACL centers. An independent Data Safety Monitoring Committee at Children's Hospital of Los Angeles monitored study progress.

\section{Eligibility}

Individual and/or parental informed consent was obtained from all subjects as per local and federal requirements. Eligible patients were $\leq 21$ years of age when originally diagnosed and $\geq 1$ year of age at relapse. They had relapsed B-precursor or T cell ALL with $\geq 25 \%$ bone marrow blasts by morphology. CNS involvement was allowed. During the phase I portion of the trial, patients were required to have at least one prior treatment attempt, with no limit placed on the number of prior treatment regimens. Patients were eligible after allogeneic stem cell transplant as long as they were not being treated actively for graft-versus-host-disease.

Karnofsky or Lansky scores $\geq 50$ for patients older than 10 years of age, or 10 years of age or younger, respectively, were required at study entry. Allergy to asparaginase products, boron or mannitol, prior exposure to $\geq 350 \mathrm{mg} / \mathrm{m}^{2}$ of anthracycline (in doxorubicin equivalent dosing), or left ventricular fractional shortening less than $30 \%$ excluded participation. Other exclusion criteria were: serum creatinine more than the upper limit of normal (ULN) for age; direct bilirubin $>1.5$ times the institutional ULN; ALT $>4$ times ULN; history of pancreatitis; serum amylase or lipase > 2 times ULN; or active uncontrolled infection. As neuropathy has been described both for bortezomib and vincristine, patients with pre-existing $\geq$ grade 2 motor or sensory neuropathy were also excluded.

\section{Treatment and Dose Escalation}

The treatment regimen is shown in Table I. Only 1 course of therapy was planned. Bortezomib dose escalation included dose levels of $1 \mathrm{mg} / \mathrm{m}^{2} /$ dose (starting dose level) and $1.3 \mathrm{mg} / \mathrm{m}^{2} /$ dose (maximum planned dose level). De-escalation of bortezomib to $0.7 \mathrm{mg} / \mathrm{m}^{2} /$ dose was planned if dose-limiting toxicity (DLT) was observed at the starting dose level. No dose escalation of the other chemotherapy agents was planned. Originally, CNS therapy included only intrathecal chemotherapy on days 1 and 15 regardless of CNS status. After four patients were enrolled and a patient with CNS involvement did not clear the CSF, weekly triple intrathecal chemotherapy was introduced for patients with CNS disease.

Patients were enrolled in groups of three patients, with dose escalation following a standard $3+3$ patient cohort escalation design [17]. Briefly, 0/3 DLTs would allow escalation, 1/3 DLTs required expanding to six patients, $1 / 6$ DLTs allowed escalation, $\geq 2$ DLTs required de-escalation, and the maximum tolerated dose (MTD) was the highest dose administered at which no more than $1 / 6$ DLTs was observed. Dose escalation was based on DLTs occurring during the single planned course of therapy.

Toxicity was graded using the CTCAE criteria, version 3.0 (http://ctep.cancer.gov). DLT was defined as any of the following toxicities deemed by the investigator as possibly, probably, or definitely attributable to bortezomib: $\geq$ grade 3 sensory neuropathy or neuropathic pain; marrow hypoplasia (less than $10 \%$ cellularity) which continued 6 weeks from the start of the course; grade 4 nonhematologic toxicity excluding infection (septic shock, typhlitis), fever and neutropenia, fatigue, electrolyte abnormalities, hyper- or hypoglycemia, nausea or vomiting, or AST, ALT, or bilirubin elevations that returned to grade 1 by the time of blood count recovery following completion of the course.

Bortezomib was immediately discontinued for any DLT. Patients could continue with the remainder of the backbone therapy excluding bortezomib. PEG-asparaginase was to be discontinued

TABLE I. Treatment Regimen

\begin{tabular}{|l|c|c|c|c|c|c|c|c|c|c|c|}
\hline \multicolumn{1}{|r}{ Day } & 1 & 2 & 4 & 8 & 11 & 14 & 15 & 18 & 22 & 29 & 36 \\
\hline Bortezomib (1 or $\left.1.3 \mathrm{mg} / \mathrm{m}^{2}\right)$ & B & & B & B & B & & & & \multicolumn{3}{|c|}{ Evaluation } \\
\hline Vincristine (1.5 $\left.\mathrm{mg} / \mathrm{m}^{2}\right)$ & V & & & V & & & V & & V & & \\
\hline Doxorubicin $\left(60 \mathrm{mg} / \mathrm{m}^{2}\right)$ & Dox & & & & & & & & & & \\
\hline Dexamethasone $\left(10 \mathrm{mg} / \mathrm{m}^{2}\right)$ & & & & & & $\rightarrow$ & & & & & \\
\hline PEG-asparaginase (2500 U/ $\left.\mathrm{m}^{2}\right)$ & & PEG & & PEG & & & PEG & & PEG & & \\
\hline Intrathecal (IT) Ara-C & & & & & & & & & & & \\
\hline CNS Negative: IT Methotrexate & & & & & & & ITM & & & & \\
\hline CNS Positive: Triple IT treatment & & & & ITT & & & ITT & & ITT & & \\
\hline
\end{tabular}


for anaphylaxis or systemic allergic reactions, symptomatic pancreatitis, significant bleeding, or significant thrombosis. No modification of PEG-asparaginase or dexamethasone for hyperglycemia was planned.

\section{Response Evaluation}

Bone marrow aspirate/biopsy and $\mathrm{CBC}$ to assess response were done on day 29. A bone marrow procedure was not required if the patient has an absolute blast count $(\mathrm{ABC})$ greater than or equal to $2,500 / \mu 1$ in the peripheral blood on day 29. If the marrow was hypoplastic and peripheral blood counts had not recovered to absolute neutrophil count (ANC) $>750 / \mu 1$ and a platelet count $>75,000 / \mu 1$, a repeat marrow aspiration and complete blood count were required weekly until recovery or progression. If the patient had $\leq 5 \%$ lymphoblasts in the bone marrow (M1 marrow) but did not achieve an ANC $>750 / \mu 1$ and a platelet count $>75,000 / \mu 1, \mathrm{CBC}$ was repeated weekly until count recovery or relapse.

The following definitions of response were used: CR-M1 ( $<5 \%$ blasts) bone marrow with no evidence of circulating blasts or extramedullary disease and with recovery of peripheral counts (absolute neutrophil count (ANC) $>750 / \mu$ and platelet count $>75,000 / \mu 1)$; CR without platelet recovery $(\mathrm{CRp})$, M1 bone marrow with no circulating blasts or extramedullary disease and recovery of ANC $>750 / \mu 1$, but insufficient recovery of platelets $(<75,000 / \mu 1)$; partial remission (PR), complete disappearance of circulating blasts and achievement of M2 (5-25\% blasts) marrow status, without new sites of extramedullary disease, and with recovery of absolute neutrophil counts (ANC $>750 / \mu 1$ ); stable disease (SD), does not satisfy the criterion for PD, or has recovery of ANC $>750 / \mu 1$ but fails to qualify for $\mathrm{CR}, \mathrm{CRp}$, or PR; progressive disease (PD), an increase of at least $25 \%$ in the absolute number of circulating leukemic cells, development of new sites of extramedullary disease, or other laboratory or clinical evidence of PD, with or without recovery of ANC or platelets. Minimal residual disease (MRD) was not evaluated as a part of this study.

\section{RESULTS}

\section{Patients and Dose Escalation}

A total of 10 patients were enrolled in the phase I portion of this study (Table II). There were two males and eight females. 18 months, one patient age was 9.8 years (range $3.3-16.4$ years). Nine patients had B-precursor ALL and one patient had T-cell ALL. Five patients were treated in first relapse, (very early relapse $<18$ months, one patient; early relapse 18-36 months, one patient; late relapse $>36$ months, three patients). Five patients were in second relapse. None of the patients had primary refractory disease, and none had prior stem-cell transplantation.

A total of four patients were treated on dose-level 1 (bortezomib $1 \mathrm{mg} / \mathrm{m}^{2} /$ dose) (Table II). The third patient enrolled at this dose level did not receive the correct doses of dexamethasone, was considered incompletely evaluable for toxicity and not evaluable for response. This patient was removed from further protocol therapy after day 8 and replaced to ensure a total of three fully evaluable patients. No DLT was seen, so escalation to dose level 2 was initiated.

A total of six patients were then enrolled into dose level 2 (bortezomib $0.3 \mathrm{mg} / \mathrm{m}^{2} /$ dose) (Table II). The first patient on this dose level suffered DLT immediately after the first dose of bortezomib. Based on the rule that 1/3 DLTs required expanding

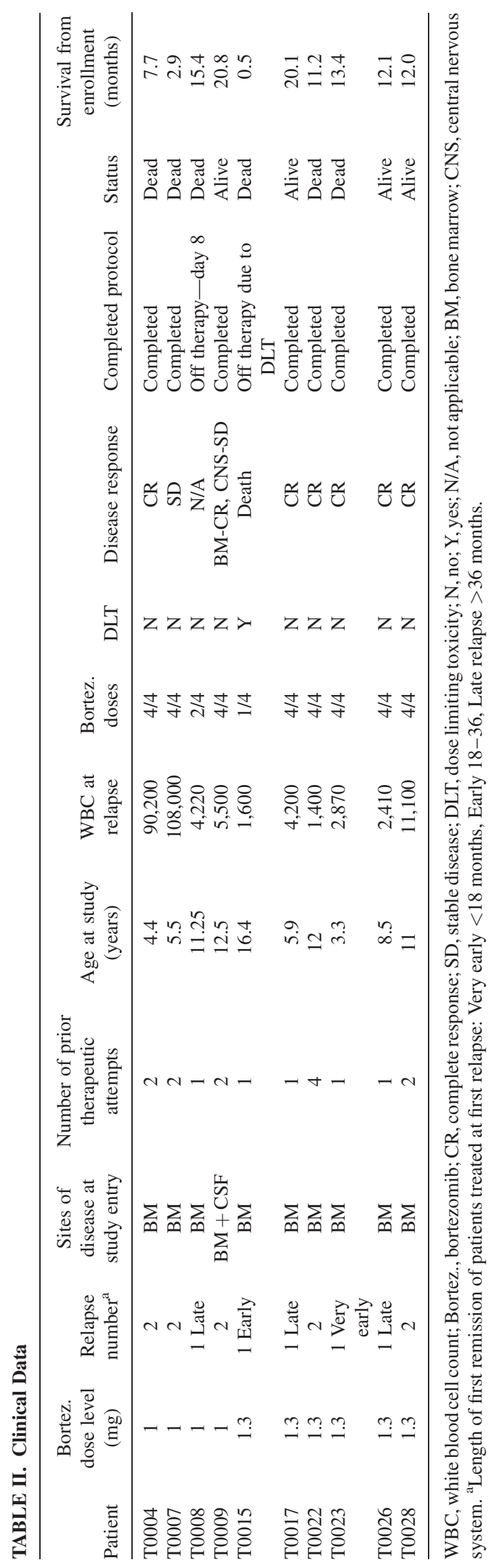


to six patients, an additional five patients were enrolled to that dose level. No further DLT was seen and dose level 2 was deemed tolerable. Since no further escalation of bortezomib was planned, the phase I portion of the study was completed and dose level 2 $\left(1.3 \mathrm{mg} / \mathrm{m}^{2} / \mathrm{dose}\right)$ was selected for further Phase II evaluation.

\section{Toxicity}

Details of the regimen-related toxicity are shown in Table III. Patient T0015 was enrolled on the study at dose level 2 $\left(1.3 \mathrm{mg} / \mathrm{m}^{2} /\right.$ day $)$. The patient had received the first dose of vincristine, bortezomib, and doxorubicin, but had not yet received dexamethasone. Seventy minutes after the bortezomib she developed rigors, pain, hypotension, hyperpyrexia, and loss of consciousness. She experienced grade 4 hypophosphatemia, and grade 3 myositis (rhabdomyolysis) with elevated creatine phosphokinase, aldolase, and myoglobinuria. The patient did not receive additional bortezomib. The patient recovered from this toxicity with the exception of continued grade 1 hypophosphatemia. This patient suffered hypophosphatemia during her original diagnosis and again at relapse, suggesting that the hypophosphatemia may not have been regimen-related. However, this constellation of symptoms was determined to be a DLT. The patient continued with the chemotherapy backbone of the regimen (excluding bortezomib) and expired from disseminated zygomyces infection on day 17 . No other DLT or regimen-associated mortality was seen on this part of the study.

As expected all patients suffered hematologic grade 4 toxicity. However, there was no prolonged bone marrow aplasia. Responding patients achieved platelet $>75,000 / \mu \mathrm{l}$ at median day 34 (range $27-$
42 ) and $\mathrm{ANC}>750 / \mu 1$ at median day 41 (range 28-43). Infectious complications included three episodes of bacteremia and one systemic fungal infection (the only death on this regimen). Notable also were electrolyte disturbances including hyponatremia (50\%), which has been described after bortezomib and vincristine. In one patient, SIADH may have been the cause of the hyponatremia. Hypokalemia and dehydration were also observed. Hypophosphatemia grade 3-4 was seen in two patients but was symptomatic only in the one patient described above, which was associated with rhabdomyolysis and subsequent mental status changes. Significant neurologic events included: confusion and myositis secondary to rhabdomyolysis from hypophosphatemia; depressed sensorium secondary to dehydration that recovered after fluids; cerebral ischemia from small acute cortical infarctions secondary to PEGasparaginase, which was then discontinued; and syncope that resolved with no intervention. Interestingly, two patients had mild peripheral neuropathy (grades 1 and 2), requiring no intervention, such as therapy interruption or dose modification. There was no discernible difference in the distribution of toxicity reporting between dose levels 1 and 2 .

\section{Efficacy}

Nine patients are evaluable for response. Six patients achieved a CR (67\%), and an additional one patient achieved a CR in the bone marrow but had persistent CNS leukemia (patient did not receive weekly intrathecal triple chemotherapy, which was introduced thereafter). Therefore, 7/9 (78\%) achieved a bone marrow CR. One patient achieved SD and another patient died on day 17 before response could be evaluated. Of patients in first relapse, 3/4 (75\%)

TABLE III. Grades 3-5 Toxicity Data in the 10 Patients Evaluable for Toxicity

\begin{tabular}{|c|c|c|c|c|c|}
\hline Group & Toxicity & Grade 3 & Grade 4 & Grade 5 & Total \\
\hline \multirow[t]{5}{*}{ Hematologic } & Hemoglobin & 6 & 1 & & 7 \\
\hline & Neutropenia & & 7 & & 7 \\
\hline & Lymphopenia & & 1 & & 1 \\
\hline & Leukopenia & 1 & 8 & & 9 \\
\hline & Thrombocytopenia & & 8 & & 8 \\
\hline \multirow[t]{4}{*}{ Infectious } & Febrile Neutropenia & 4 & & & 4 \\
\hline & Implant site Infection & 1 & & & 1 \\
\hline & Infection with neutropenia & 3 & & 1 & 4 \\
\hline & Pyrexia & 1 & & & 1 \\
\hline \multirow[t]{6}{*}{ Laboratory } & Hyperglycemia & 3 & & & 3 \\
\hline & Hypoalbuminemia & 1 & & & 1 \\
\hline & Hypocalcemia & & 1 & & 1 \\
\hline & Hypokalemia & 2 & 1 & & 3 \\
\hline & Hyponatremia & 5 & & & 5 \\
\hline & Hypophosphatemia & 1 & 1 & & 2 \\
\hline \multirow[t]{5}{*}{ Neurologic } & Confusion and myositis & 1 & & & 1 \\
\hline & Depressed Sensorium & 1 & & & 1 \\
\hline & Cerebral ischemia & 1 & & & 1 \\
\hline & Myalgia & 1 & & & 1 \\
\hline & Syncope & 1 & & & 1 \\
\hline \multirow[t]{3}{*}{ Hepatic/pancreas } & ALT & 3 & & & 3 \\
\hline & AST & 1 & & & 1 \\
\hline & Lipase & 1 & & & 1 \\
\hline Coagulation & PT, PTT & 3 & & & 3 \\
\hline Cardiovascular & Prolonged QT interval & 1 & & & 1 \\
\hline \multirow{2}{*}{ Gastrointestinal } & Abdominal pain & 1 & & & 1 \\
\hline & Caecitis (typhlitis) & 1 & & & 1 \\
\hline
\end{tabular}


TABLE IV. Response

\begin{tabular}{|c|c|c|c|c|c|c|}
\hline & $\mathrm{n}$ & Not evaluable & Evaluable & $\mathrm{CR}$ & $\begin{array}{c}\text { Marrow } \\
\text { CR }\end{array}$ & $\begin{array}{c}\text { Death or no } \\
\text { response }\end{array}$ \\
\hline First relapse $^{a}$ & 5 & 1 & 4 & $375 \%$ & $375 \%$ & $1^{\mathrm{b}}$ \\
\hline Second relapse & 5 & 0 & 5 & $360 \%$ & $480 \%$ & $1^{\mathrm{c}}$ \\
\hline Total & 10 & 1 & 9 & $667 \%$ & $778 \%$ & 2 \\
\hline
\end{tabular}

${ }^{a}$ Response of patients in first relapse: Very early-1/1 CR; early: death before response assessed; late: 2/2 CR of assessable patients; ${ }^{\mathrm{b}}$ Death on day 17 before response assessed; ${ }^{\mathrm{c}}$ Stable disease.

had bone marrow CR, and of patients in second relapse 4/5 (80\%) had bone marrow CR (Table IV). This level of response, met criteria to proceed to the phase II part of this study, which is accruing patients at this time.

\section{DISCUSSION}

This phase I study in childhood ALL was designed to evaluate whether combining bortezomib with a previously active chemotherapy backbone is tolerable. It demonstrated that a standard dose of bortezomib $1.3 \mathrm{mg} / \mathrm{m}^{2}$ given on days $1,4,8$, and 11 can be safely combined with an intensive 4-drug reinduction regimen in children with relapsed ALL. Since the phase I pediatric study of single agent bortezomib established $1.3 \mathrm{mg} / \mathrm{m}^{2}$ as the MTD [13], we opted for no further dose escalation in the combination trial. Similarly, in adults with AML, bortezomib $1.5 \mathrm{mg} / \mathrm{m}^{2}$ on the same schedule was safely combined with idarubicin and cytosine arabinoside [16].

There was no prolonged bone marrow suppression from this regimen, even though thrombocytopenia is well recognized after bortezomib administration. As previously described [3], infections are common with a similar chemotherapy backbone and can lead to death from fungal infection. Although both bortezomib and vincristine can induce peripheral neuropathy only two patients suffered grade 1-2 transient peripheral neuropathy. However, four significant neurologic events (confusion, depressed sensorium, cerebral ischemia, and syncope, Table III) were ascribed to the other chemotherapy agents. Patients on the phase II portion of the study receive close monitoring for neurologic toxicity. Pulmonary toxicity, described after bortezomib use in adults [18], was not observed in this study.

Interestingly, three of four patients in first relapse achieved a complete response (CR), but more significantly, four of five in second relapse achieved complete bone marrow response (Table IV). This is a higher response rate than the $44 \% \mathrm{CR}$ after second relapse reported by Ko et al. [4] Determining remission reinduction rates in an expanded number of patients in second or above relapse is the primary objective of the phase II part of the study. Although early, this level of response is impressive because very limited single agent bortezomib activity was seen in leukemia in adults [12] and children [13]. If confirmed in the phase II part of this study, it may corroborate the in vitro synergistic activity with dexamethasone [14] as well as the clinical activity previously observed in one patient [15].

The phase II of this combination is accruing patients with greater than or equal to second relapse and will include the cohort of patients in second relapse reported here. Additionally, the data reported here were used by the COG to initiate another phase II study of bortezomib with 4-drug induction in early first bone marrow relapse.
The mechanism by which proteasome inhibition leads to increased sensitivity to chemotherapeutic agents is not clear. It was previously shown that inhibition of $\mathrm{NF}-\kappa \mathrm{B}$, which is constitutively elevated in leukemia cells, enhances the sensitivity of AML cells to chemotherapeutic agents [19]. In contrast, inhibition of NF- $\mathrm{KB}$ expression did not enhance chemosensitivity of ALL cell-lines [20]. Whether bortezomib-induced inhibition of NF- $\mathrm{KB}$ is the primary mechanism inducing leukemia cell death in vivo is uncertain. In the single agent trial of bortezomib, Horton et al. [13] observed increased NF- $\mathrm{KB}$ expression $6-12 \mathrm{hr}$ after bortezomib exposure, followed by a 4-6-fold decrease in $\mathrm{NF}-\kappa \mathrm{B}$ expression $24 \mathrm{hr}$ after bortezomib exposure. In contrast, Attar et al. reported in vivo reduction of NF- $\mathrm{KB}$ expression within $1 \mathrm{hr}$ after bortezomib [16]. Bortezomib was shown to down-regulate $\mathrm{I} \kappa \mathrm{B}$ expression and trigger NF- $\kappa \mathrm{B}$ activation in multiple myeloma, raising questions about the prevailing model that bortezomib is an $\mathrm{NF}-\kappa \mathrm{B}$ inhibitor [21]. Others describe that proteasome inhibition results in accumulation of Bax and Bim pro-apoptotic proteins, which sensitize leukemia cells to anthracycline-induced apoptosis [22]. Elucidation of the specific pathways involved may allow for additional targeted agents to be used in combination with bortezomib in the hope of sparing some of the chemotherapy agents used in the current regimen.

In summary, this phase I study of bortezomib given in standard twice-weekly doses of $1.3 \mathrm{mg} / \mathrm{m}^{2} /$ dose, combined with vincristine, dexamethasone, PEG-asparaginase, and doxorubicin is tolerable and shows promising activity in relapsed childhood ALL.

\section{REFERENCES}

1. Nguyen K, Devidas M, Cheng SC, et al. Factors influencing survival after relapse from acute lymphoblastic leukemia: A Children's Oncology Group study. Leukemia 2008;22:21422150 .

2. Gaynon PS, Harris RE, Altman AJ, et al. Bone marrow transplantation versus prolonged intensive chemotherapy for children with acute lymphoblastic leukemia and an initial bone marrow relapse within 12 months of the completion of primary therapy: Children's Oncology Group study CCG- 1941. J Clin Oncol 2006; 24:3150-3156.

3. Raetz EA, Borowitz MJ, Devidas M, et al. Reinduction platform for children with first marrow relapse of acute lymphoblastic Leukemia: A children's oncology group study[corrected]. J Clin Oncol 2008;26:3971-3978.

4. Ko RH, Ji L, Barnette P, et al. Outcome of patients treated for relapsed or refractory Acute Lymphoblastic Leukemia (ALL) - A Therapeutic Advances in Childhood Leukemia (TACL) consortium study. J Clin Oncol 2010; 28:648-654.

5. Adams J. Proteasome inhibition in cancer: Development of PS-341. Semin Oncol 2001;28:613-619. 
6. Rajkumar SV, Richardson PG, Hideshima T, et al. Proteasome inhibition as a novel therapeutic target in human cancer. J Clin Oncol 2005;23:630-639.

7. Landis-Piwowar KR, Milacic V, Chen D, et al. The proteasome as a potential target for novel anticancer drugs and chemosensitizers. Drug Resist Updat 2006;9:263-273.

8. Schenkein D. Proteasome inhibitors in the treatment of B-cell malignancies. Clin Lymphoma 2002;3:49-55.

9. Vink J, Cloos J, Kaspers GJ. Proteasome inhibition as novel treatment strategy in leukaemia. Br J Haematol 2006;134:253262.

10. Kane RC, Bross PF, Farrell AT, et al. Velcade: U. S. FDA approval for the treatment of multiple myeloma progressing on prior therapy. Oncologist 2003;8:508-513.

11. Houghton PJ, Morton CL, Kolb EA, et al. Initial testing (stage 1) of the proteasome inhibitor bortezomib by the pediatric preclinical testing program. Pediatr Blood Cancer 2008;50:37-45.

12. Cortes J, Thomas D, Koller C, et al. Phase I study of bortezomib in refractory or relapsed acute leukemias. Clin Cancer Res 2004;10: 3371-3376.

13. Horton TM, Pati D, Plon SE, et al. A phase 1 study of the proteasome inhibitor bortezomib in pediatric patients with refractory leukemia: A children's oncology group study. Clin Cancer Res 2007;13:1516-1522.

14. Horton TM, Gannavarapu A, Blaney SM, et al. Bortezomib interactions with chemotherapy agents in acute leukemia in vitro. Cancer Chemother Pharmacol 2006;58:13-23.
15. Brown RE, Bostrom B, Zhang PL. Morphoproteomics and bortezomib/dexamethasone-induced response in relapsed acute lymphoblastic leukemia. Ann Clin Lab Sci 2004;34:203-205.

16. Attar EC, De Angelo DJ, Supko JG, et al. Phase I and pharmacokinetic study of bortezomib in combination with idarubicin and cytarabine in patients with acute myelogenous leukemia. Clin Cancer Res 2008;14:1446-1454.

17. Ahn C. An evaluation of phase I cancer clinical trial designs. Stat Med 1998; 17:1537-1549.

18. Miyakoshi S, Kami M, Yuji K, et al. Severe pulmonary complications in Japanese patients after bortezomib treatment for refractory multiple myeloma. Blood 2006;107:34923494.

19. Romano MF, Petrella A, Bisogni R, et al. Effect of NF-kappaB/Rel inhibition on spontaneous vs chemotherapy-induced apoptosis in AML and normal cord blood CD34+ cells. Leukemia 2003;17: $1190-1192$

20. Min DJ, Moskowitz NP, Brownstein C, et al. Diverse pathways mediate chemotherapy-induced cell death in acute lymphoblastic leukemia cell lines. Apoptosis 2006;11:1977-1986.

21. Hideshima T, Ikeda H, Chauhan D, et al. Bortezomib induces canonical nuclear factor- $\{$ kappa $\} \mathrm{B}$ activation in multiple myeloma cells. Blood 2009;114:1046-1052

22. Pigneux A, Mahon FX, Moreau-Gaudry F, et al. Proteasome inhibition specifically sensitizes leukemic cells to anthracyclininduced apoptosis through the accumulation of Bim and Bax proapoptotic proteins. Cancer Biol Ther 2007;6:603-611. 